\title{
Ion irradiation of asphaltite as an analogue of solid hydrocarbons in the interstellar medium
}

\author{
G. Strazzulla ${ }^{1}$ and L. Moroz ${ }^{2}$ \\ 1 INAF - Osservatorio Astrofisico di Catania, via Santa Sofia 78, 95123 Catania, Italy \\ e-mail: gianni@ct.astro.it \\ 2 German Aerospace Center (DLR), Institute of Planetary Research, Rutherfordstr. 2, 12489 Berlin, Germany
}

Received 2 December 2004 / Accepted 21 December 2004

\begin{abstract}
We present the results of experiments of low temperature ion irradiation of pure asphaltite samples and those covered with water ice layers. Asphaltite (a natural bitumen) was used as an analogue of carbonaceous interstellar grains on which waterrich icy mantles can be formed in the dense and cold phases of the interstellar medium. We simulated irradiation by cosmic ions in the laboratory to study, by in situ infrared spectroscopy, the formation of molecular species in the asphaltite samples and at the interface between the refractory solid (asphaltite) and the water ice mantle. New molecular species were formed at the expense of water molecules and atoms (mainly carbon and hydrogen) from the asphaltite substrate. The vibration modes we detected at low temperature $(16 \mathrm{~K}$ ) include: $\mathrm{C}-\mathrm{H}$ stretch (carbynoids), $\mathrm{C}-\mathrm{H}$ asymmetric stretch (acetylene), $\mathrm{C}-\mathrm{H}$ aromatic stretch, $\mathrm{C}=\mathrm{O}$ stretch in $\mathrm{CO}_{2}, \mathrm{C} \equiv \mathrm{O}$ stretch in $\mathrm{CO}$, and $\mathrm{C} \equiv \mathrm{C}$ stretch (carbynoids). Some of these species remain stable after warming of the irradiated samples to room temperature.
\end{abstract}

Key words. astrochemistry - methods: laboratory - techniques: spectroscopic - ISM: molecules - dust, extinction

\section{Introduction}

Carbonaceous dust is formed in circumstellar regions of C-rich stars and ejected in the interstellar medium. Spatially resolved $3 \mu \mathrm{m}$ spectroscopy of proto-planetary nebulae revealed that roto-vibrational bands of acetylene $\left(\mathrm{C}_{2} \mathrm{H}_{2}\right)$ and hydrogen cyanide $(\mathrm{HCN})$ are concentrated in a compact region near the central star (Goto et al. 2003). The 3.3 and $3.4 \mu \mathrm{m}$ emission bands of aromatic and aliphatic hydrocarbons are detected far from the star. The intensity of aliphatic emission bands relative to the aromatic ones decreases with distance from the central star. The spatial separation between gas and dust has been interpreted as evidence that small molecules $\left(\mathrm{C}_{2} \mathrm{H}_{2}\right.$ and $\left.\mathrm{HCN}\right)$ are the parents of the dust (Goto et al. 2003). Although the dust composition and structural properties are not well known, the shapes and relative intensities of the 3.3 and $3.4 \mu \mathrm{m}$ emission features are well reproduced by a thermally annealed quenched carbonaceous composite (Goto et al. 2000).

In evolved objects such as CRL 618, a post-AGB star (Chiar et al. 1998), and in the diffuse interstellar medium (e.g. Pendleton \& Allamandola 2002, and references therein) absorption by carbonaceous dust is different: the aromatic $3.3 \mu \mathrm{m}$ feature is not observed and the aliphatic $3.4 \mu \mathrm{m}$ feature has a different shape. The shape of this feature has been successfully modelled by Mennella and co-workers (Mennella et al. 1999, 2001, 2002, 2003; Munoz-Caro et al. 2001). Those authors, based on the results of laboratory experiments, proposed that energetic processing (ion irradiation and/or
UV photolysis) of carbonaceous materials (mostly hydrogenated amorphous carbon) effectively destroys $\mathrm{C}-\mathrm{H}$ bonds. On the other hand, $\mathrm{sp}^{2}$ hybridized $\mathrm{C}-\mathrm{H}$ bonds are newly formed by exposing amorphous carbons to an atomic hydrogen flux. The activated aliphatic band well reproduces the feature observed in the diffuse interstellar medium where the activation process dominates. In a dense medium, icy mantles accrete and protect the core from exposure to atomic hydrogen. This explains why the $3.4 \mu \mathrm{m}$ feature is not observed in dense clouds and challenges a previous hypothesis (e.g., Greenberg et al. 1995) that it originates from energetic processing of icy mantles in dense clouds.

From the above we can infer that carbonaceous material, freshly ejected in the interstellar medium (ISM) from circumstellar regions of dust-producing stars, is a complex mixture of aliphatic and aromatic compounds, although the latter are not observed in the ISM dust. However aromatic components are almost certainly present since they are likely to be expelled from evolved stars and polycyclic aromatic hydrocarbons (PAHs) are observed, in emission, as an essential constituent of the gas phase (e.g., Allamandola et al. 1989; Puget \& Leger 1989). In dense molecular clouds most of the gas phase species are rapidly condensed on refractory cores, forming icy mantles that very likely contain condensed aromatic hydrocarbons. The frozen mantles are rich in water, the dominant ice in the Universe, that was identified as a component of dust grains a long time ago (Willner et al. 1982; Smith et al. 1989). 
In the light of these facts, studies of energetic processing of aliphatic and aromatic solid hydrocarbons and their mixtures with water (or other ices) are extremely relevant both to astrochemistry and to astrobiology. Mennella and coworkers (Mennella et al. 1999, 2001, 2002, 2003; Munoz-Caro et al. 2001) have studied processing of aliphatic amorphous hydrocarbons covered with water ice mantles. Some studies have also been devoted to energetic processing of PAHs mixed with water ice. Bernstein et al. (1999, 2003) demonstrated that UV photolysis and proton irradiation of PAHs mixed with ices produce, by side group addition, alcohols, quinones and ethers that might have important implications for extraterrestrial biogenesis. Tuleta et al. (2001) outlined the bioastrophysical aspects of low energy $\left(3.5 \mathrm{keV} \mathrm{He}^{+}\right)$ion irradiation of frozen anthracene containing water confirming the formation of quinones. Mahajan et al. (2003) observed formation of carboncarbon bonds after photochemical alkylation of PAHs and alkanes in solution, in thin films in contact with liquid water and in icy matrices.

In this paper we present new experimental results on ion irradiation $\left(30 \mathrm{keV} \mathrm{He}^{+}\right)$of a solid bitumen, asphaltite, both pure and covered with water ice layers. Here asphaltite is used as an analogue of a complex carbonaceous material having both aliphatic and aromatic bonds. The technique used to reveal the irradiation-induced effects is IR transmittance spectroscopy in the $3700-1900 \mathrm{~cm}^{-1}(2.7-5.26 \mu \mathrm{m})$ range. Other spectral ranges, in particular between 2000 and $1250 \mathrm{~cm}^{-1}(5-8 \mu \mathrm{m})$, would be equally relevant in view of the comparison with observations of the diffuse ISM (Pendleton \& Allamandola 2002): future studies will be devoted to different spectral ranges and detailed comparison with astronomical observations. The only previous experiments on ion irradiation of asphaltite were recently performed by Moroz et al. (2004) who irradiated bulk samples of pure asphaltite and studied their spectral reflectance in the $0.3-2.5 \mu \mathrm{m}$ range. Asphaltite is very dark in the visual region and has red-sloped spectra in the visible and nearinfrared ranges. It may be a good analogue for refractory organic solids on the surfaces of primitive objects from the outer solar system (Moroz et al. 1998). The results by Moroz et al. (2004) show that irradiation-induced carbonization gradually neutralizes the spectral slopes of the red organic solids. This implies a similar space weathering trend for the surfaces of airless bodies optically dominated by spectrally red organic components.

\section{Some properties of asphaltite}

An asphaltite (gilsonite) sample from Izhem, Timano-Pechora Basin, Russia, was provided by Moscow State University. Moroz et al. (1998) analyzed the sample for C, H, N, O, and $\mathrm{S}$ content, and characterized it with $\mathrm{X}$-ray diffractometry (XRD), solid-state ${ }^{13} \mathrm{C}$ nuclear magnetic resonance (NMR), and reflectance spectroscopy. In this study, we used asphaltite sample \#3 from Moroz et al. (1998). The asphaltite was composed of $81.6 \mathrm{wt} . \% \mathrm{C}, 9.5 \mathrm{wt} \% \mathrm{H}, 6.4 \mathrm{wt} \% \mathrm{~S}, 1.3 \mathrm{wt} . \% \mathrm{O}$, and 1.1 wt. $\% \mathrm{~N}$, normalized to $100 \mathrm{wt}$. $\%$. Its atomic ratios of $\mathrm{H} / \mathrm{C}$ and $\mathrm{O} / \mathrm{C}$ were 1.4 and 0.012 respectively (Moroz et al. 1998). The XRD showed an average spacing between aromatic layers

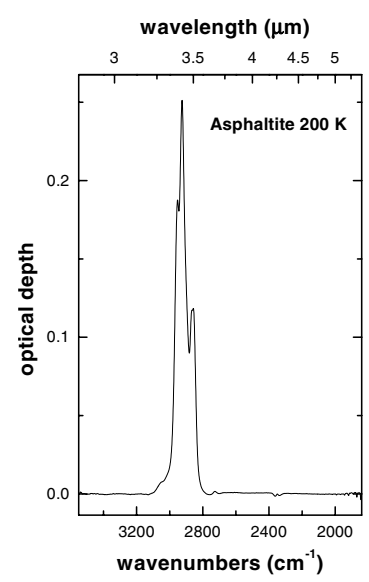

Fig. 1. IR trasmittance spectrum of asphaltite films deposited on a silicon substrate. The spectral range is $3700-1900 \mathrm{~cm}^{-1}(2.7-5.26 \mu \mathrm{m})$. The band peak positions and assignment are given in Table 1 .

Table 1. IR bands of asphaltite in the $3700-1900 \mathrm{~cm}^{-1}(2.7-5.26 \mu \mathrm{m})$ range.

\begin{tabular}{lll}
\hline \hline $\begin{array}{c}\text { Asphaltite } 200 \mathrm{~K} \\
\text { Peak position } \\
\mathrm{cm}^{-1}\end{array}$ & $\mu \mathrm{m}$ & Assignment \\
\hline 3050 & 3.28 & $\mathrm{C}-\mathrm{H}$ aromatic str \\
2952 & 3.39 & aliphatic $\mathrm{CH}_{3}$ asym str \\
2925 & 3.42 & aliphatic $\mathrm{CH}_{2}$ asym str \\
2857 & 3.5 & aliphatic $\mathrm{CH}_{2}$ sym str \\
2727 & 3.67 & aldehyde $(\mathrm{RCHO})$ \\
\hline
\end{tabular}

d002 of $3.65 \AA$. The average thicknesses of aromatic crystallites $L_{\mathrm{c}}$ for asphaltite were $6.8 \AA$. The carbon aromaticity $f_{\mathrm{a}}$ (the fraction of aromatic carbon) derived from ${ }^{13} \mathrm{C}$ NMR was 0.335 . Asphaltite has a density of about $1 \mathrm{~g} \mathrm{~cm}^{-3}$ (Uspensky et al. 1964; Klubov 1983).

Compared to most other solid oil bitumens, asphaltite is rich in $\mathrm{H}$ and has a relatively disordered structure. Its polycyclic aromatic structural units are relatively small. Therefore, it is rather bright and transparent in the infrared due to a large optical gap $E_{\mathrm{g}}$ (the energy gap between valence and conduction bands; McMichael et al. 1954). Asphaltite is very dark in the visible range due to a broad complex electronic absorption feature typical of PAHs (Brown 1955; McMichael et al. 1954; Painter et al. 1985; Ito et al. 1988). This feature is centred in the UV, while its long-wavelength wing causes a steep positive (red) spectral slope in the visible and near-infrared.

Asphaltite exhibits several vibrational absorption bands mostly due to aliphatic and aromatic functional groups. The spectral range investigated here (see Fig. 1) is $3700-1900 \mathrm{~cm}^{-1}$ (2.7-5.26 $\mu \mathrm{m})$. In this range, the most prominent bands are due to the stretching (3.4-3.5 $\mu \mathrm{m})$ vibrations of alkyl $\mathrm{CH}_{2}$ and $\mathrm{CH}_{3}$ groups. In addition, an absorption band due to aromatic $\mathrm{C}-\mathrm{H}$ stretch $(3.3 \mu \mathrm{m})$ is seen as a shoulder in Fig. 1. The band peak positions are given in Table 1 .

\section{Experimental procedures}

To prepare asphaltite samples suitable for ion irradiation and in situ transmittance IR spectroscopy, we poured some drops of 
asphaltite dissolved in chloroform on an IR-transparent Si substrate. By evaporation of the chloroform, uniform asphaltite films were formed on the substrate. The thickness of the films was about $1 \mu \mathrm{m}$, as evaluated using an optical microscope. The infrared transmittance spectra were obtained in a highvacuum chamber $\left(P<10^{-7}\right.$ mbar $)$ interfaced with, through IR-transparent windows, a FTIR spectrophotometer (Bruker Equinox 55). Samples were in thermal contact with a closedcycle helium cryostat whose temperature can be varied between 10 and $300 \mathrm{~K}$. The vacuum chamber was interfaced with an ion implanter (200 kV, Danfysik 1080-200) which generates ions with energies up to $200 \mathrm{keV}$ (400 keV for double ionization). The ion beam produces a spot greater than the area probed by the infrared beam. In this work we used $30 \mathrm{keV} \mathrm{He}^{+}$ions. In order to avoid macroscopic heating of the target the current density was maintained in the range of $100 \mathrm{nA} \mathrm{cm}^{-2}$ to a few $\mu \mathrm{A} \mathrm{cm}^{-2}$. The ion fluence in ions $\mathrm{cm}^{-2}$ is measured by a charge integrator from the ion current monitored during irradiation. The substrate plane is placed at an angle of 45 degrees with respect to the IR beam and the ion beam so that spectra can be taken in situ, even during irradiation, without tilting the sample. Spectra can be taken at selected temperatures in the range of 10-300 K. All the spectra shown below were taken with a resolution of $4 \mathrm{~cm}^{-1}$ using a DTGS detector. In some experiments water vapour was admitted into the chamber by a needle valve to deposit water ice layers on the asphaltite films. Ice deposition occurred at $16 \mathrm{~K}$ and at a rate of about $2 \AA \mathrm{s}^{-1}$. The deposition time was about $5 \mathrm{~min}$. In this way we obtained water ice films with a thickness of about $600 \AA$.

$30 \mathrm{keV} \mathrm{He}{ }^{+}$ions penetrate about $3000 \AA$ in asphaltite calculated using the TRIM program (Transport of Ions in Matter; e.g., Ziegler 1977; Ziegler et al. 1996) and about $4000 \AA$ in water ice. Thus the asphaltite samples are thick targets where ions are stopped and remain implanted. The water ice films are thinner than the penetration depth of incoming ions.

For more details on the experimental set up see Strazzulla et al. (2001).

\section{Results}

Figure 2 shows two spectra of asphaltite taken at $200 \mathrm{~K}$ before (dotted line) and after (solid line) ion irradiation $\left(1.8 \times 10^{16}\right.$ $30 \mathrm{keV} \mathrm{He} \mathrm{cm}^{-2}$ ). After the collection of the spectrum of the non-irradiated sample (dotted line in Fig. 2) a new background was taken that includes the sample itself. In this way, after irradiation, newly formed bands are seen as having positive optical depths. Bands with negative optical depths are those of asphaltite that is progressively destroyed by ion irradiation. This experiment was performed at $200 \mathrm{~K}$ to avoid any contamination from water vapour which could be present in the background vacuum.

The first two columns in Table 2 list the peak positions $\left(\mathrm{cm}^{-1}\right.$ and $\left.\mu \mathrm{m}\right)$ of the newly formed bands. Their assignments are given in the last column. The most intense bands are those at $3301 \mathrm{~cm}^{-1}(3.03 \mu \mathrm{m})$ and $2106 \mathrm{~cm}^{-1}(4.75 \mu \mathrm{m})$ that are attributed to $\mathrm{C}-\mathrm{H}$ stretch in carbynoid structures $\left(\mathrm{R}-(\mathrm{C} \equiv \mathrm{C})_{m}-\mathrm{H}\right.$; odd ending polyynes $)$ and to $(\mathrm{C} \equiv \mathrm{C})$ stretch,

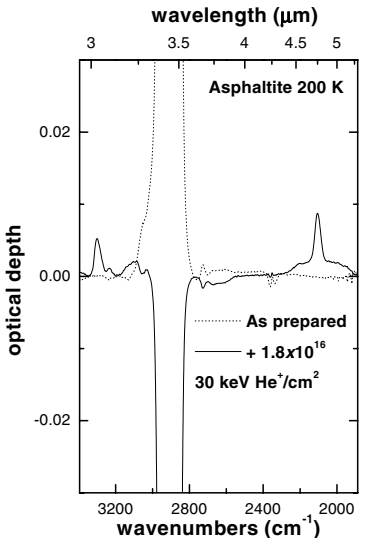

Fig. 2. IR spectra of asphaltite taken at $200 \mathrm{~K}$ before (dotted line) and after (solid line) ion irradiation $\left(1.8 \times 10^{16} 30 \mathrm{keV} \mathrm{He} \mathrm{cm}^{-2}\right)$. The spectrum of the non-irradiated sample (dotted line) was measured including the Si substrate in the background; the spectrum of the irradiated sample (solid line) was measured including the non-irradiated sample in the background. Thus newly formed bands are seen as having positive optical depths; bands with negative optical depths are those of the asphaltite that is progressively destroyed.

respectively (Strazzulla et al. 2004). Also clearly detectable are bands at $3236 \mathrm{~cm}^{-1}(3.09 \mu \mathrm{m})$ due to the $\mathrm{C}-\mathrm{H}$ stretch in acetylenes $\left(\mathrm{H}-(\mathrm{C} \equiv \mathrm{C})_{m}-\mathrm{H}\right)$, and $3086 \mathrm{~cm}^{-1}(3.24 \mu \mathrm{m})$ due to the aromatic $\mathrm{C}-\mathrm{H}$ stretch.

Figure 3 shows the spectra (in two spectral regions, note the different scales) of water ice deposited on an asphaltite sample at $16 \mathrm{~K}$, dotted line; of the same sample after ion irradiation $30 \mathrm{keV} \mathrm{He}^{+}\left(1.5 \times 10^{16} \mathrm{~cm}^{-2}\right)$, dashed line; and after warming the target to $200 \mathrm{~K}$, solid line. The thickness of the deposited water ice layer was about $1.8 \times 10^{17} \mathrm{H}_{2} \mathrm{O}$ molecules $\mathrm{cm}^{-2}$ (about $550 \AA$ ), i.e. lower than the ion penetration depth.

The spectrum of asphaltite does not change with the temperature and is identical to that shown in Fig. 1, except that the sample irradiated at $16 \mathrm{~K}$ was somewhat thicker (see below). Water ice exhibits, in the investigated spectral range, a fundamental band at $3280 \mathrm{~cm}^{-1}(3.05 \mu \mathrm{m})$ due to the $\mathrm{O}-\mathrm{H}$ vibrations (symmetric and antisymmetric) and a combination band at about $2200 \mathrm{~cm}^{-1}(4.54 \mu \mathrm{m})$ that is scarcely visible in Fig. 3. Upon irradiation the intensity of the water ice bands decreases due to sputtering and chemical alteration.

In this case also the background was taken before ice deposition and includes the asphaltite sample. Thus, the dotted line exhibits only the water ice bands. During irradiation asphaltite is dehydrogenated (bands due to original $\mathrm{C}-\mathrm{H}$ stretches appear as having negative optical depths) and new bands are formed. Columns three and four in Table 2 list the peak positions $\left(\mathrm{cm}^{-1}\right.$ and $\mu \mathrm{m}$ ) of the newly formed bands. Their assignments are given in the last column. In addition to the bands seen after irradiation of the pure asphaltite sample, formation of $\mathrm{CO}_{2}$ and $\mathrm{CO}$ is evident from the detection of the bands at $2341 \mathrm{~cm}^{-1}$ $(4.27 \mu \mathrm{m})$ and $2135 \mathrm{~cm}^{-1}(4.68 \mu \mathrm{m})$, respectively. In addition, a band at $1956 \mathrm{~cm}^{-1}(5.11 \mu \mathrm{m})$ appears that could be due to cumulene $(C=C) n$ bonds (e.g., Kavan \& Kastner 1999).

After irradiation, the sample was warmed and the spectrum at $200 \mathrm{~K}$ is also shown in Fig. 3 (solid line). The spectrum does 
Table 2. Peak positions of newly formed IR bands in the 3700-1900 $\mathrm{cm}^{-1}(2.7-5.26 \mu \mathrm{m})$ region after irradiation of asphaltite containing samples with $30 \mathrm{keV} \mathrm{He}^{+}$ions.

\begin{tabular}{|c|c|c|c|c|c|c|}
\hline \multicolumn{2}{|c|}{ Asphaltite $200 \mathrm{~K}$} & \multicolumn{2}{|c|}{$\mathrm{H}_{2} \mathrm{O}$ on asphaltite $16 \mathrm{~K}$} & \multicolumn{2}{|c|}{ Residue $200 \mathrm{~K}$} & \multirow[t]{2}{*}{ Assignment } \\
\hline $\mathrm{cm}^{-1}$ & $\mu \mathrm{m}$ & $\mathrm{cm}^{-1}$ & $\mu \mathrm{m}$ & $\mathrm{cm}^{-1}$ & $\mu \mathrm{m}$ & \\
\hline 3301 & 3.03 & 3286 & 3.04 & 3297 & 3.03 & $\mathrm{R}-(\mathrm{C} \equiv \mathrm{C})_{m}-\mathrm{H}$ str (carbynoids) \\
\hline 3236 & 3.09 & 3233 & 3.09 & - & - & $\mathrm{C}-\mathrm{H}$ asym str (acetylene) \\
\hline 3086 & 3.24 & 3086 & 3.24 & 3076 & 3.25 & $\mathrm{C}-\mathrm{H}$ aromatic str \\
\hline- & - & 2341 & 4.27 & - & - & $\mathrm{C}=\mathrm{O}$ str in $\mathrm{CO}_{2}$ \\
\hline- & - & 2135 & 4.68 & - & - & $\mathrm{C} \equiv \mathrm{O}$ str in $\mathrm{CO}$ \\
\hline 2106 & 4.75 & 2114 & 4.73 & 2112 & 4.73 & $\mathrm{C} \equiv \mathrm{C}$ str \\
\hline- & - & 1956 & 5.11 & 1954 & 5.12 & $(\mathrm{C}=\mathrm{C}) \mathrm{n} ?$ \\
\hline
\end{tabular}

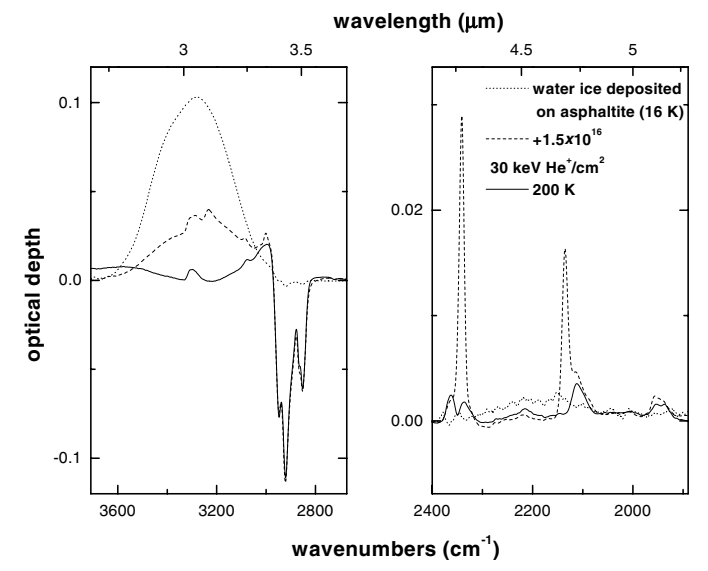

Fig. 3. Spectra (in two spectral regions, note the different scales) of water ice deposited on an asphaltite sample at $16 \mathrm{~K}$, dotted line; of the same sample but after ion irradiation $\left(30 \mathrm{keV} \mathrm{He}+1.5 \times 10^{16} \mathrm{~cm}^{-2}\right)$, dashed line; and after warming the target to $200 \mathrm{~K}$, solid line. The background was taken before ice deposition and includes the asphaltite sample.

not change up to room temperature. The bands due to volatile species disappear because of sublimation and those of a refractory material remain. The peak positions of the latter bands are also reported in Table 2.

The fact that the original asphaltite sample, pure or covered with ice layers, is chemically modified by ion irradiation is supported by additional evidence. Pure asphaltite samples are soluble in chloroform. After ion bombardment, a fraction of the irradiated material becomes insoluble. This fraction increases with increasing ion fluence but has not been quantitatively measured.

The integrated intensity $\left(\mathrm{cm}^{-1}\right)$ of the IR bands is proportional to the column density (molecules $\mathrm{cm}^{-2}$ ) of the responsible species through the integrated absorbance $\left(\mathrm{cm} \mathrm{molecule}{ }^{-1}\right.$ ) of that particular vibration. Figures 4 and 5 show the integrated intensities (band areas) of some bands versus ion fluences for the experiments with pure asphaltite and with asphaltite/ice, respectively. Integrated absorbance values are known only for a few bands, therefore here we present only the band areas.

Figure 4, left panel, demonstrates the decrease of the area of the complex $\mathrm{C}-\mathrm{H}$ stretching bands of asphaltite with increasing

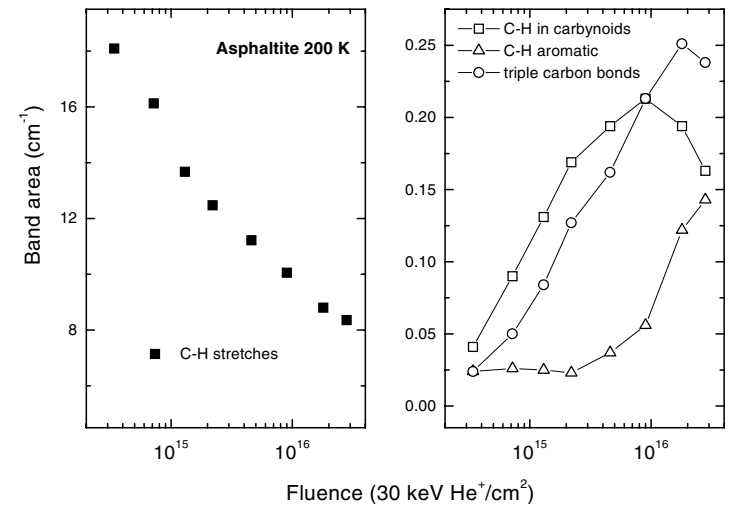

Fig. 4. Areas of the complex asphaltite $\mathrm{C}-\mathrm{H}$ bands versus ion fluence (left panel); areas of the newly formed bands vs. ion fluence are shown in the right panel.

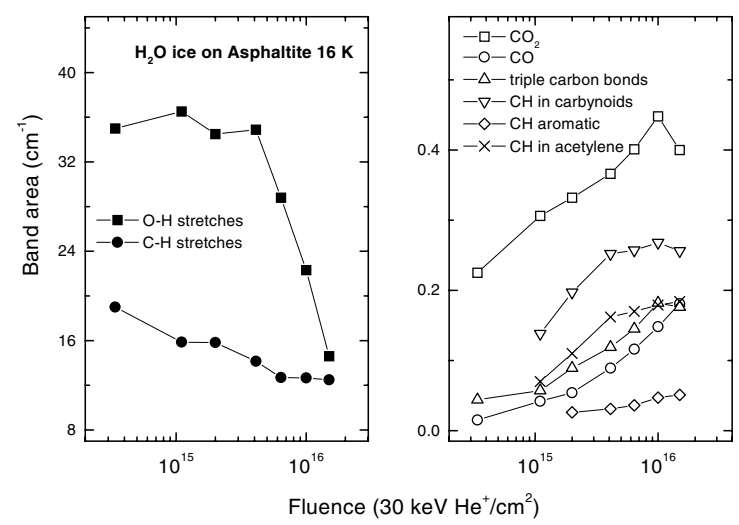

Fig. 5. Areas of the complex C-H stretching bands of asphaltite, and of the $\mathrm{O}-\mathrm{H}$ stretching band in water ice deposited on asphaltite as a function of ion fluence (left panel); areas of the newly formed bands versus ion fluence are shown in the right panel.

ion fluence. The area is calculated from 2995 to $2815 \mathrm{~cm}^{-1}$ and exhibits three distinct peaks (see Table 1 and Fig. 1). The initial band area was about $22 \mathrm{~cm}^{-1}$, while at the end of irradiation it drops to $8.2 \mathrm{~cm}^{-1}$, being reduced to about $38 \%$ of the initial value. The reduction is essentially due to a loss of hydrogen and does not reach zero since the ions we used do not penetrate through the whole asphaltite layer. The right panel of Fig. 4 shows the areas of the newly formed bands as a function of 
ion fluence. Early formation of carbynoids is evident from the appearance of the carbon-hydrogen and triple carbon bonds. Aromatic C-H bands at $3086 \mathrm{~cm}^{-1}(3.24 \mu \mathrm{m})$ appear after a threshold of about $3 \times 10^{15} 30 \mathrm{keV} \mathrm{He}^{+} \mathrm{cm}^{-2}$.

Figure 5, left panel, shows the band areas versus ion fluence for the complex $\mathrm{C}-\mathrm{H}$ stretches of asphaltite (circles) and $\mathrm{O}-\mathrm{H}$ stretches (symmetric and anti-symmetric) in the water ice deposited on top (squares). Due to loss of hydrogen the asphaltite $\mathrm{C}-\mathrm{H}$ band area is reduced to $60 \%$ of the initial value. The area of the water ice $\mathrm{O}-\mathrm{H}$ band exhibits different behaviour: no initial $\mathrm{H}_{2} \mathrm{O}$ loss at low irradiation fluences. This is due to the variation of the integrated absorbance of the band induced by ion irradiation. Leto \& Baratta (2003) have shown that the integrated absorbance of the $\mathrm{O}-\mathrm{H}$ stretches in water ice undergoes a $10 \%$ increase at low ion fluences because of a rearrangement of the amorphous structure of the ice. The behaviour observed in Fig. 5 is probably due to the competition between the increase of the integrated absorbance and the destruction process of the water ice layer.

\section{Discussion}

Evolution of solids (ices, silicates, and carbonaceous phases) in the circumstellar and interstellar media is governed by a number of processes such as surface reactions, UV photolysis, particle irradiation and thermal annealing. Here we simulated one such process, namely ion irradiation of a hydrocarbon target (asphaltite) as an analogue for a complex material produced in carbon-rich stars and expelled into the interstellar medium. Irradiation in circumstellar environments or in the diffuse interstellar medium was simulated by irradiating pure asphaltite layers at $200 \mathrm{~K}$. Calculations indicated that in the diffuse interstellar medium the temperature of the dust is on the order of $10 \mathrm{~K}$ (Greenberg 1971; Mathis et al. 1983). However the gas density is such that icy mantles cannot be formed. Occasionally diffuse clouds contract to form dense molecular clouds where icy layers are formed on the grains. The dense cloud phase was simulated in experiments of ion irradiation of water ice layers deposited at low $T$ on top of the asphaltite. After irradiation warming the target causes the sublimation of volatile molecules and this simulates further passage of the dust in the diffuse medium.

The flux of low energy cosmic rays irradiating grains in the interstellar medium is not well known. A reasonable estimate was given by Moore (1999). She calculated that in cold dense clouds, ions deposit about $30 \mathrm{eV} /$ molecule (in $10^{8}$ years), a factor of 10 less than in the diffuse medium. Our experiments were conducted with fluences of $30 \mathrm{keV} \mathrm{He}$ up to $3 \times 10^{16} \mathrm{~cm}^{-2}$ with a maximum of energy deposition of about $400 \mathrm{eV} / \mathrm{C}$ atom in the penetrated layers. Such a range of energy deposition (0-400 eV/C-atom) covers those expected in the dense and in the diffuse interstellar medium. Furthermore, the dominant contribution of cosmic rays comes mainly from low energy protons in the $\mathrm{MeV}$ range, which lose energy mainly through ionizations and excitations of the target atoms as $30 \mathrm{keV} \mathrm{He}^{+}$ions do. This justifies the extrapolation of our results to the astrophysical scenario.
It is interesting to note that after irradiation of asphaltite samples, both pure and covered with water ice, carbon bonds characteristic of linear chains are formed (carbynoids and cumulenes); new aromatic bonds are also evident. All these bonds are also present after warming the low $T$ samples. The band centered near $3300 \mathrm{~cm}^{-1}(3.03 \mu \mathrm{m})$ has also been detected after irradiation of solid acetylene (Strazzulla et al. 2002), solid benzene and cluster-assembled carbon thin films (Strazzulla \& Baratta 1991; Strazzulla et al. 2004). Thus its formation seems to be typical of many irradiated hydrogen-bearing carbonaceous materials. The band centered near $2110 \mathrm{~cm}^{-1}(4.73 \mu \mathrm{m})$ is even more common, being produced also after irradiation of hydrogenated amorphous carbon (Mennella et al. 2004). Formation of the latter two bands in circumstellar environments, in dense and diffuse clouds is likely. The band at $3300 \mathrm{~cm}^{-1}(3.03 \mu \mathrm{m})$ would be difficult to observe in dense clouds since it overlaps with a very intense water ice feature. It is more likely to be detected in circumstellar or diffuse environments. Broad features currently attributed to the overlapping $\mathrm{C}_{2} \mathrm{H}_{2}$ and $\mathrm{HCN}$ absorption bands have been observed in the same spectral range in the circumstellar regions of C-rich objects (Chiar et al. 1998; Cernicharo et al. 1999; Goto et al. 2003; Gauba \& Parhasarathy 2004). We suggest that those broad features could, at least in part, be due to a solid state feature similar to the one observed in our study. To our knowledge, there has not been any report of a band near $2110 \mathrm{~cm}^{-1}(4.73 \mu \mathrm{m})$ in both diffuse and dense clouds: it should be searched for (see also Mennella et al. 2004).

Another feature produced in our experiments is due to aromatic C-H stretching vibrations near $3080 \mathrm{~cm}^{-1}(3.25 \mu \mathrm{m})$. Brooke et al. (1999) reported observations of a band centered at $3.25 \mu \mathrm{m}$ towards several young stellar objects.

In addition, in our experiments we observed the formation of carbon mono- and dioxide at the ice/asphaltite interface. This result also is quite general and occurs in many cases when water ice is deposited on solid carbonaceous materials, such as organic residues from irradiation of methane-rich (Strazzulla \& Palumbo 1997) or benzene-rich (Gomis et al. 2004) frozen mixtures and amorphous carbons (Mennella et al. 2004). Based on their laboratory results, Mennella et al. (2004) demonstrated that energetic processing in dense clouds does not dominate other $\mathrm{CO}_{2}$ formation processes, but contributes to other processes occurring on ice mantles. The spectral profile and the contribution to the observed column densities make solid CO formed by cosmic-ray irradiation a good candidate for the red component of the interstellar CO stretching feature (Mennella et al. 2004).

Gomis et al. (2004) suggest that the cosmic-ray irradiation may account for the quantity of $\mathrm{CO}_{2}$ ice detected on the surfaces of the Galilean satellites where $\mathrm{CO}_{2}$ has been detected mainly trapped in the non-ice material, rather than in the pure water ice.

Acknowledgements. We thank Dr. Greg Michael for helpful comments and suggestions. This research has been supported by the Italian Space Agency (ASI), and the Italian Ministero dell'Istruzione, Università e Ricerca (MIUR). 


\section{References}

Allamandola, L. J., Tielens, G. G. M., \& Barker, J. R. 1989, ApJS, 71, 733

Bernstein, M. P., Sandford, S. A., Allamandola, L. J., et al. 1999, Science, 283, 1135

Bernstein, M. P., Moore, M. H., Elsila, J. E., et al. 2003, ApJ, 582, L25

Brooke, T. Y., Sellgren, K., \& Geballe, T. R. 1999, ApJ, 517, 883

Brown, J. K. 1955, J. Chem. Soc., March 1995, 744

Cernicharo, J., Yamamura, I., Gonzalez-Alfonso, E., et al. 1999, ApJ, 526, L41

Chiar, J. E., Pendleton, Y. J., Geballe, T. R., \& Tielens, A. G. G. M. 1998, ApJ, 507, 281

Gauba, G., \& Parthasarathy, M. 2004, A\&A, 417, 201

Gomis, G., \& Strazzulla, G. 2004, Icarus, submitted

Goto, M., Maihara, T., Terada, H., et al. 2000, A\&A, 141, 149

Goto, M., Gaessler, W., Hayano, Yutaka, et al. 2003, ApJ, 589, 419

Greenberg, J., Mayo 1971, A\&A, 12, 240

Greenberg, J., Mayo, Li, Aigen, Mendoza-Gomez, et al. 1995, ApJ, 455, L177

Ito, O., Seki, H., \& Iino, M. 1988, Fuel, 67, 573

Kavan, L., \& Kastner, J. 1999, in Carbyne and Carbynoid Structures (Dordrecht: Kluver Academic Publisher), 343

Klubov, B. 1983, Natural Bitumens of the North (Moscow: Nauka), USSR, in Russian

Leto, G., \& Baratta, G. A. 2003, A\&A, 397, 7

Mahajan, T. B., Elsila, J. E., Deamer, D. W., \& Zare, R. N. 2003, Origins of Life and Evolution of the Biosphere, 33, 17

Mathis, J. S., Mezger, P. G., \& Panagia, N. 1983, A\&A, 128, 212

McMichael, B. D., Kmetko, E. A., \& Mrozowski, S. 1954, J. Opt. Soc. Am., 44, 26

Mennella, V., Brucato, J. R., Colangeli, L., \& Palumbo, P. 1999, ApJ, 524, L71

Mennella, V., Munoz Caro, G. M., Ruiterkamp, R., et al. 2001, A\&A, 367,355

Mennella, V., Brucato, J. R., Colangeli, L., \& Palumbo, P. 2002, ApJ, 569,531

Mennella, V., Baratta, G. A., Esposito, A., Ferini, G., \& Pendleton, Y. J. 2003, ApJ, 587, 727
Mennella, V., Palumbo, M. E., \& Baratta, G. A. 2004, ApJ, 615, 1073

Moore, M. H. 1999, The Physics and Chemistry of Ice in the Interstellar Medium, in Solid Interstellar Matter: the ISO Revolution, ed. L. d'Hendecourt, C. Joblin, \& A. Jones (Berlin: Springer-Verlag), 199

Moroz, L. V., Arnold, G., Korochantsev, A. V., \& Wasch, R. 1998, Icarus, 134, 253

Moroz, L., Baratta, G., Strazzulla, G., et al. 2004, Icarus, 170, 214

Munoz Caro, G. M., Ruiterkamp, R., Schutte, W. A., Greenberg, J. M., \& Mennella, V. 2001, A\&A, 367, 347

Painter, P., Starsinic, M., \& Coleman, M. 1985, in ed. J. R. Ferraro, \& L. J. Basile, Fourier Transform Infrared Spectroscopy: Application to Chemical Systems (London: Academic Press), 4, 169

Pendleton, Y. J., \& Allamandola, L. J. 2002, ApJS, 138, 75

Puget, J. L., \& Leger, A. 1989, ARA\&A, 27, 161

Smith, R. G., Sellgren, K., \& Tokunaga, A. T. 1989, ApJ, 344, 413

Strazzulla, G., \& Baratta, G. A. 1991, A\&A, 241, 310

Strazzulla, G., \& Palumbo, M. E. 1997, in Proc. First ISO Workshop on Analytical Spectroscopy, ESA SP 419, 11

Strazzulla, G., Baratta, G. A., \& Palumbo, M. E. 2001, Spectrochim. Acta A, 57, 825

Strazzulla, G., Baratta, G. A., Domingo, M., \& Satorre, M. A. 2002, NIM B, 191, 714

Strazzulla, G., Baratta, G. A., Battiato, S., \& Compagnini, G. 2004, in Polyynes: Synthesis, Properties, and Applications, ed. F. Cataldo (New York: Marcel Dekker), in press

Tuleta, M., Gabla, L., \& Madej, J. 2001, Phys. Rev. Lett., 87, id. 078103

Uspensky, V. A., Radchenko, O. K., Glebovskaya, E. A., et al. 1964, Basics of Genetic Classification of Bitumens (Leningrad: Nedra), USSR, in Russian

Willner, S. P., Gillett, F. C., Herter, T. L., et al. 1982, ApJ, 253, 174

Ziegler, J. F. 1977, The Stopping and Range of Ions in Matter. V. 2-6 (New York: Pergamon Press)

Ziegler, J. F., Biersack, J. P., \& Littmark, U. 1996, The Stopping and Range of Ions in Solids (New York: Pergamon Press) 